\title{
Correction to: Genome-wide assessment of population structure and genetic diversity of Eucalyptus urophylla based on a multi-species single-nucleotide polymorphism chip analysis
}

Huixiao Yang ${ }^{1} \cdot$ Fang $\mathrm{Xu}^{1} \cdot$ Huanqin Liao ${ }^{1} \cdot$ Weihua Zhang ${ }^{1} \cdot$ XiaoHui Yang ${ }^{1} \cdot$ Bin $\mathrm{Xu}^{1} \cdot$ Wen Pan ${ }^{1}$

Published online: 3 June 2020

(C) Springer-Verlag GmbH Germany, part of Springer Nature 2020

Correction to: Tree Genetics \& Genomes (2020) 16:39 https://doi.org/10.1007/s11295-020-1422-x

The correct Author list is presented in this paper.

Huixiao Yang, Fang Xu and Huanqin Liao contributed equally to this work.

The online version of the original article can be found at https://doi.org/ $10.1007 / \mathrm{s} 11295-020-1422-\mathrm{x}$

\section{XiaoHui Yang}

xiaohuiyang@sinogaf.cn

$\triangle \quad$ Wen Pan

Panwen@sinogaf.cn

1 Guangdong Provincial Key Laboratory of Silviculture, Protection and Utilization/Guangdong Academy of Forestry,

Guangzhou 510520, People's Republic of China 